\title{
Formate Dehydrogenase Molybdenum and Tungsten Sites-Observation by EXAFS of Structural Differences
}

\author{
S. P. Cramer, C-L. Liu, L. E. Mortenson
}

Corporate Research-Science Laboratories, Exxon Research and Engineering Company Annandale, New Jersey

\section{J. T. Spence}

Department of Chemistry, Utah State University, Logan, Utah

\author{
S-M. Liu, I. Yamamoto, and L. G. Ljungdahl \\ Department of Biochemistry, University of Georgia, Athens, Georgia
}

\begin{abstract}
Preliminary EXAFS data has been collected on the molybdenum ( $K$-edge) in $C$. pasteurianum formate dehydrogenase and the tungsten ( $L_{\mathrm{III}}$-edge) in $C$. thermoaceticum formate dehydrogenase. In the presence of dithionite, the tungsten enzyme was devoid of $\mathrm{W}=\mathrm{O}$ bonds, and exhibited average $\mathrm{W}-(\mathrm{O}, \mathrm{N})$ and $\mathrm{W}-\mathrm{S}$ bond lengths of $2.13 \pm 0.03 \AA$ and $2.39 \pm 0.03 \AA$, respectively. In sharp contrast, the $C$. pasteurianum molybdenum site has three $M o=O$ bonds with an average bond length of $1.74 \pm 0.03 \AA$. It is also the first molybdenum enzyme found lacking Mo-S bonds, and does not appear to be redox active in the presence of formate or dithionite. Model compounds $\mathrm{WO}_{2}(8 \text {-hydroxyquinoline })_{2}=$ $\mathrm{WO}_{2}(\mathrm{ox})_{2}$, and $\mathrm{WO}_{2}(8 \text { mercaptoquinoline })_{2}=\mathrm{WO}_{2}(\text { tox })_{2}$, were also examined. Respective predicted bond lengths for $\mathrm{WO}_{2}(\mathrm{ox})_{2}$ and $\mathrm{WO}_{2}$ (tox $)_{2}$ were $\mathrm{W}=\mathrm{O}$ of $1.71,1.73 \AA ; \mathrm{W}-\mathrm{N}$ of $2.31,2.29 \AA ; W-O$ or $W-S$ of 1.92 or $2.40 \AA$, with estimated uncertainties of $\pm 0.03 \AA$.
\end{abstract}

The enzymes which catalyze the reversible oxidation of formate to carbon dioxide, formate dehydrogenases, exhibit substantial diversity in their prosthetic group composition and reactivity with various electron acceptors [1, 2]. The prosthetic

Address reprint requests to Dr. S. P. Cramer, Corporate Research-Science Laboratories, Clinton Township, Route 22 East, Annandale, NJ 08801. 
groups of these enzymes can include $\mathrm{Fe} / \mathrm{S}$ clusters, molybdenum, tungsten, and/or selenium. As part of a program to characterize systematic trends in the structure of molybdenum and tungsten enzymes [3-8], the molybdenum center in $C$. pasteurianum formate dehydrogenase, CP-FD, a Mo, Fe/S protein, has been examined by $\mathrm{x}$-ray absorption spectroscopy [8], and compared with the tungsten site of $C$. thermoaceticum formate dehydrogenase, CT-FD, [9], a W, Se, Fe/S protein. The results show that the molybdenum and tungsten environments could hardly be more different.

CT-FD was purified to a specific activity of $486 \mathrm{units} / \mathrm{mg}$ by previously described procedures [9] and concentrated to $180 \mathrm{mg} / \mathrm{ml}$ with an Amicon PM-30 membrane. The activity was measured by monitoring the formation of NADPH [10]. Samples which were $2 \mathrm{mM}$ in sodium dithionite were loaded anaerobically into Lucite cuvettes, and the spectra were recorded at room temperature. Although the activity was low on a protein basis, this was caused by the presence of tungsten-free enzyme. After correction for the presence of apoprotein it was estimated that better than $95 \%$ of the tungsten-containing sites were active. Assays done immediately after data collection showed $8 \%$ loss of activity.

CP-FD was purified to a specific activity of $254 \mathrm{units} / \mathrm{mg}$ by an adaptation of previously described procedures $[11,12]$ and concentrated to $42 \mathrm{mg} / \mathrm{ml}$ with an Amicon XM-100A membrane in pH $850 \mathrm{mM}$ Tris $\mathrm{HCl}$ buffer. The sample "asisolated" contained no reductants, and had a volume of $1.5 \mathrm{ml}$. The "formatereduced" enzyme was prepared by addition of $20 \mu$ moles of formate. Subsequent addition of $12 \mu$ moles of dithionite produced the "dithionite-reduced" sample. The model compounds $\mathrm{WO}_{2}$ (8-hydroxyquinoline $)_{2}$ and $\mathrm{WO}_{2}$ (8-mercaptoquinoline $)_{2}$ were prepared using literature procedures [13].

The EXAFS data were recorded on several occasions at the Stanford Synchrotron Radiation Laboratory using filtered fluorescence detection techniques [14], and processed and analyzed according to previously described procedures [15, 16]. To surmount a severe "glitch" problem with the Si[2,2,0] crystal in the W $L_{\text {III }}$ region, a weighted average with a comparable spectrum obtained on a $\mathrm{Si}[1,1,1]$ crystal was generated, with the $k$-dependent weighting of the $[2,2,0]$ contribution adjusted smoothly to zero in the glitch regions.

The molybdenum $K$ absorption edge region of "as-isolated" CP-FD in Figure 1 shows a strong peak at $20008 \mathrm{eV}$ which is a bound state transition characteristic of terminal oxo groups $[5,17]$. The major inflection point position is in a region characteristic of Mo(VI) with oxygen and/or nitrogen ligands [3]. The "formatereduced" spectrum shows a small shift to lower energy, but the overall shape is identical to the "as-isolated" spectrum. Because the monochromator makes large $1-\mathrm{eV}$ steps in this region, the apparent shift is within experimental error, and does not necessarily indicate binding of formate to molybdenum. It is certainly too small to indicate reduction of the molybdenum. However, the rapid color change demonstrated reduction of the $\mathrm{Fe} / \mathrm{S}$ centers. Addition of dithionite, in order to generate more reducing conditions, yielded an edge indistinguishable from the "as-isolated"' spectrum.

The CP-FD EXAFS Fourier transform, Figure 2, has a strong low $R$ peak with an amplitude corresponding to $3 \mathrm{Mo}=\mathrm{O}$ bonds. In contrast with all other 


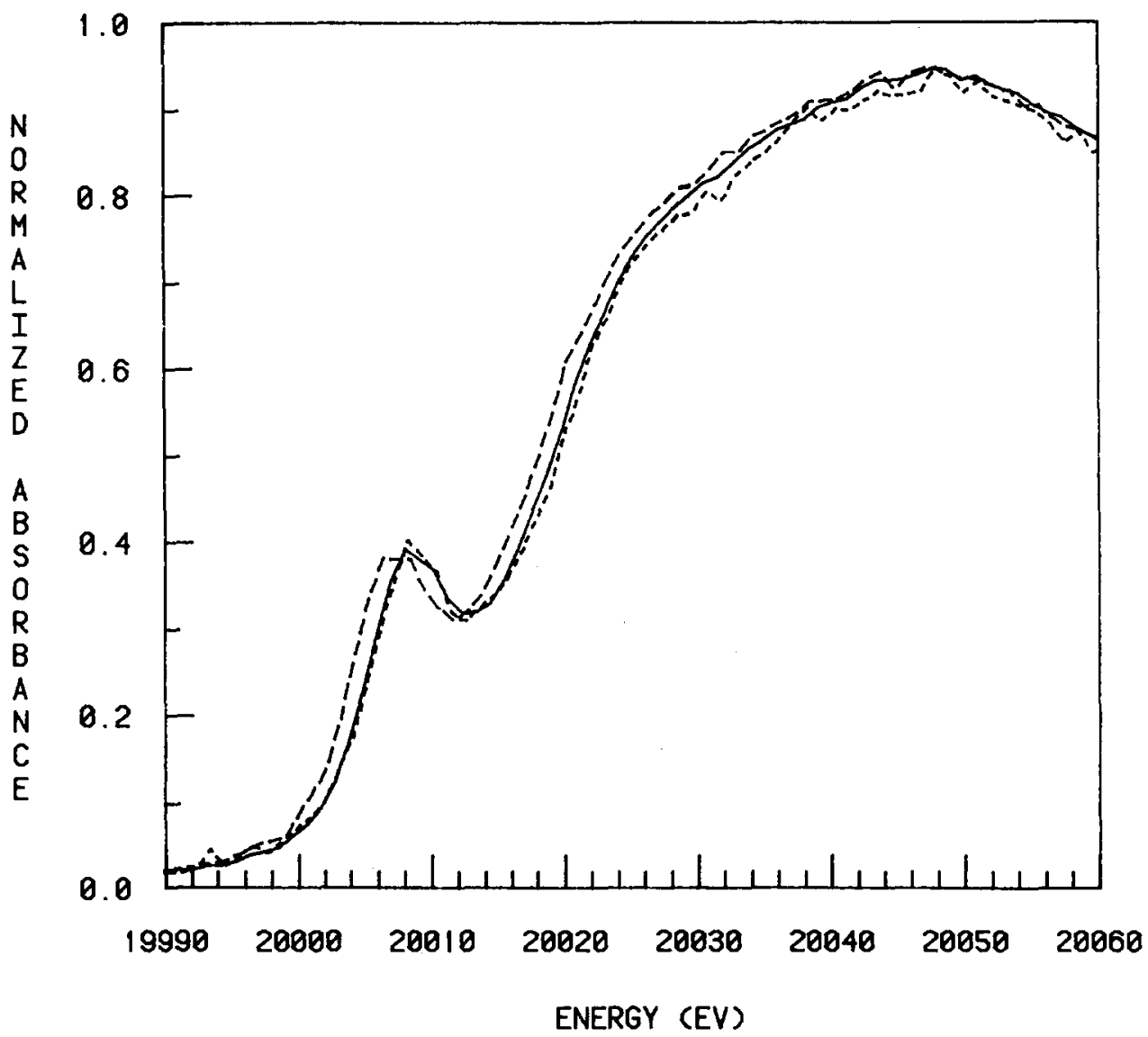

FIGURE 1. Molybdenum x-ray absorption edges for Clostridium pasteurianum formate dehydrogenase. Calibration assumed $20003.9 \mathrm{eV}$ for molybdenum foil first inflection point. ___, Enzyme as-isolated; - - , formate-reduced enzyme; --- dithionite-treated enzyme.

molybdenum enzymes, there is no significant longer distance peak that would indicate a Mo-S interaction. Rather, there are only weak features barely above the truncation ripple; these are probably from Mo-O and/or Mo-N interactions.

The transform of the CT-FD EXAFS exhibits a single broad and asymmetric peak in the region where normal W-OR and W-SR features occur. Furthermore, it lacks any evidence for a short $\mathrm{W}=\mathrm{O}$ bond. As illustrated in Figure 2, the spectrum of CT-FD is vastly different from that of CP-FD, as well as from two dioxotungsten "models." The $\mathrm{WO}_{2}(\mathrm{ox})_{2}$ and $\mathrm{WO}_{2}(\mathrm{tox})_{2}$ models show twin peak patterns, similar to those previously observed for dioxo-molybdenum models [15]. The first peaks correspond to the $\mathrm{W}=\mathrm{O}$ interactions, while the second peaks represent the combination of $\mathrm{W}-\mathrm{N}$ and $\mathrm{W}-\mathrm{O}$ or $\mathrm{W}-\mathrm{S}$ interactions.

Curve-fitting the Fourier-filtered EXAFS data, as summarized in Scheme 1, allows more precise estimation of the metal-ligand distances. The structures of the 


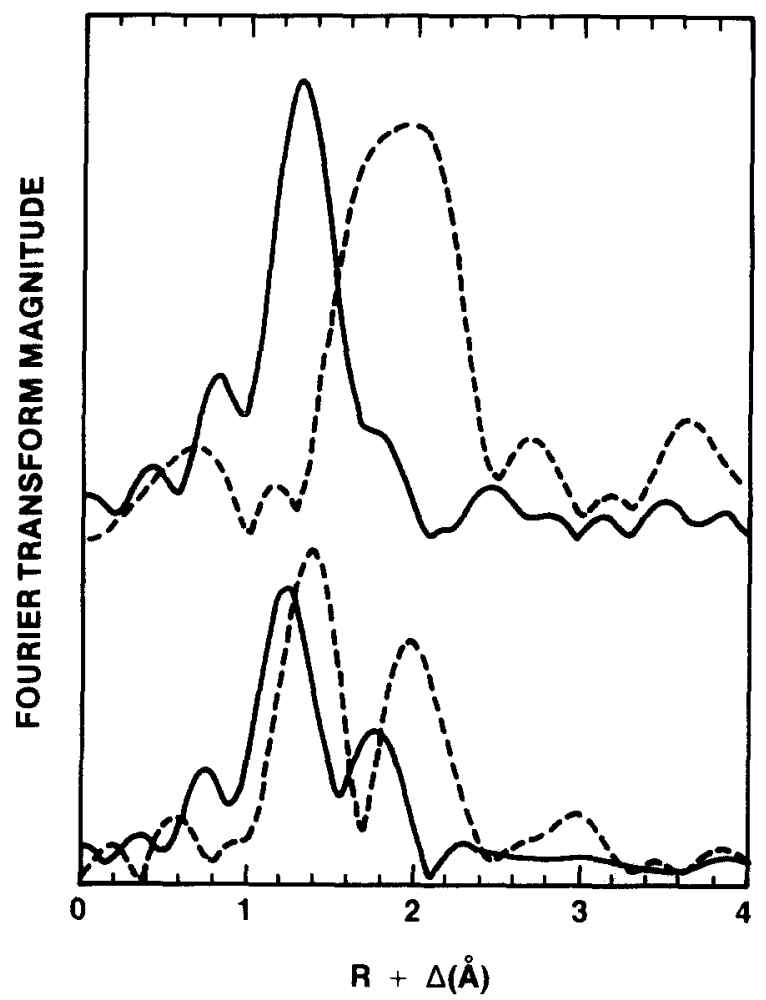

FIGURE 2. EXAFS Fourier transforms for formate dehydrogenases and tungsten model compounds. Top: ___ Clostridium pasteurianum formate dehydrogenase; - - - Clostridium thermoaceticum formate dehydrogenase. Bottom: ——_, $\mathrm{WO}_{2}(\mathrm{ox})_{2} ;-\cdots, \mathrm{WO}_{2}(\mathrm{tox})_{2}$. Transform range: $k=4$ $12 \AA^{-1}, k^{3}$ weighting.

tungsten model compounds were derived from the EXAFS bond lengths combined with the assumption of $c i s-\mathrm{W}=\mathrm{O}$ bonds and equivalent ligands.

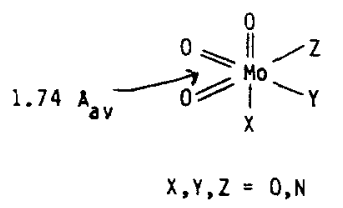

C. pasteurianum

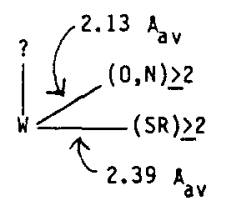

C. thermoaceticum
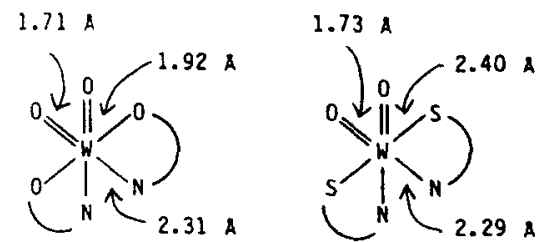

$\mathrm{WO}_{2}(\mathrm{Ox})_{2}$

$\mathrm{WO}_{2}(\mathrm{tox})_{2}$

SCHEME 1. Proposed structures for enzyme metal sites and tungsten model compounds

The $1.74 \AA \mathrm{Mo}=\mathrm{O}$ bond length found by curve-fitting the CP-FD EXAFS is longer than the 1.66-1.72 $\AA$ values found for other molybdenum enzymes. This is consistent with the proposed tri-oxo structure, since tri-oxo bonds tend to be longer than mono- or dioxo-Mo $=\mathrm{O}$ bonds, but shorter than the 1.77- $\AA$ bonds in $\mathrm{MoO}_{4}{ }^{2-}$. Two additional different $\mathrm{Mo}-\mathrm{O}, \mathrm{N}$ distances were required to completely fit the CP-CR data, but the nature of these ligands remains unclear. 
The CT-FD fits indicate $\mathrm{W}-\mathrm{O}, \mathrm{N}$ and $\mathrm{W}-\mathrm{S}$ interactions at 2.13 and $2.39 \AA$, respectively. However, there is a large uncertainty in the coordination numbers. EXAFS amplitude corrections may be required, because the enzyme data were recorded in the fluorescence mode using filters, while the model compound spectra were recorded in transmission mode. Additional uncertainty stems from the possbile presence of a W-Se interaction, since inclusion of a W-Se interaction at $2.6 \AA$ does improve the fit and lower the number of $\mathrm{W}-\mathrm{S}$ interactions required. Selenium EXAFS experiments are planned to resolve this point. The proposed tungsten environment bears some resemblance to the structure previously reported for $E$. coli nitrate reductase [7].

A tungsten cofactor can be obtained from CT-FD [18], which has excitation and emission spectra very similar to the degraded molybdenum cofactor from sulfite oxidase, nitrate reductase, and xanthine oxidase [19, 20]. A similar fluorescent material can also be extracted from CP-FD [21]. Johnson and Rajagopalan [19] have proposed that the molybdenum cofactor contains a novel 6-substituted pterin with two sulfurs in the side chain and that molybdenum is coordinated by these sulfurs. The CT-FD tungsten EXAFS, together with the results of fluorescence excitation and emission spectroscopy [18], are consistent with the presence of a similarly substituted pterin for the tungsten cofactor.

The absence of sulfur ligands indicated by the EXAFS studies of CP-FD indicates differences between its molybdenum environment and the model proposed by Johnson and Rajagopalan [19]. These results suggest that either the same pterin binds metals in different ways in different enzymes, that different types of pterins are present in different molybdenum enzymes, or that the cofactor acutally binds to molybdenum through nitrogen and/or oxygen ligands, with the sulfur ligands frequently observed by EXAFS being provided by the protein. Although the possibility remains that sulfur ligands interact with CP-FD molybdenum under other conditions, the current EXAFS results nonetheless clearly show that molybdenum can be bound to proteins in a sulfur-free environment.

The staff of SSRL is thanked for their assistance. Work done at SSRL was supported by the Department of Energy, Office of Basic Energy Sciences, the National Science Foundation, Division of Materials Research, and the National Institute of Health, Biotechnology Resources Program, Division of Research Resources. The worked performed at the University of Georgia was supported by Public health Service grant No. AM-27323 from the National Institute of Arthritis, Diabetes, Digestive and Kidney Diseases, and Project DE-ASO-79 ER 10499 from the United States Department of Energy.

\section{REFERENCES}

1. L. G. Ljungdahl, in Molybdenum and Molybdenum-containing Enzymes, M. P. Coughlan, Ed, Pergamon Press, New York, 1980, pp. 463-486.

2. L. G. Ljungdahl and H. G. Wood, in $B_{12}$, D. Dolphin, Ed., Vol. 2, John Wiley and Sons, New York, 1982, pp. 165-202.

3. S. P. Cramer, K. O. Hodgson, W. O. Gillum, and L. E. Mortenson, J. Am. Chem. Soc. 100, 3398-3407 (1978).

4. S. P. Cramer, W. O. Gillum, K. O. Hodgson, L. E. Mortenson, E. I. Stiefel, J. R. Chisnell, W. J. Brill, and V. K. Shah, J. Am. Chem. Soc. 100, 3814-3819 (1978).

5. S. P. Cramer, R. Wahl, and K. V. Rajagopalan, J. Am. Chem. Soc. 103, 7721-7727 (1981).

6. S. P. Cramer, J. G. G. Moura, A. V. Xavier, and J. LeGall, J. Inorg. Biochem. 20, 275-280 (1984). 
7. S. P. Cramer, L. S. Solomonson, M. W. W. Adams, and L. E. Mortenson, J. Am. Chem. Soc. 106, 1467-1471 (1984).

8. S. P. Cramer in Advances in Inorganic and Bioinorganic Mechanisms, A. G. Sykes, Ed, Academic Press, London, 1983.

9. I. Yamamoto, T. Saiki, S. M. Liu, and L. G. Ljungdahl, J. Biol. Chem. 258, 1826-1832 (1983).

10. L. G. Ljungdahl and J. R. Andreesen, Methods Enzymol. 53, 360-372 (1978).

11. C.-L. Liu and L. E. Mortenson, J. Bacteriol, 159, 375-380 (1984).

12. P. A. Scherer and R. K. Thauer, Eur. J. Biochem. 85, 125-135 (1978).

13. C. A. Rice, P. M. H. Kroneck, and J. Spence, Inorg. Chem. 20, 1996-2000 (1981).

14. S. P. Cramer and R. A. Scott, Rev. Sci. Instrum. 52, 395-399 (1981).

15. S. P. Cramer, K. O. Hodgson, E. I. Stiefel, and W. E. Newton, J. Am. Chem. Soc. 100, 27482761 (1978).

16. S. P. Cramer, EXAFS of Inorganic Systems, C. D. Garner and S. S. Hasnain, Eds., Daresbury Laboratory, Daresbury, England (1981), pp. 47-50.

17. F. W. Kutzler, R. A. Scott, J. M. Berg, K. O. Hodgson, S. Doniach, S. P. Cramer, and C. H. Chang, J. Am. Chem. Soc. 103, 6083-6088 (1981).

18. K. V. Rajagopalan, personal communication.

19. J. L. Johnson and K. V. Rajagopalan, Proc. Natl. Acad. Sci. U.S.A. 79, 6856-6860 (1982).

20. J. L. Johnson, B. E. Hainline, and K. V. Rajagopalan, J. Biol. Chem. 255, 1783-1786 (1980).

21. C.-L. Liu and L. E. Mortenson, unpublished results.

Received December 7, 1983; accepted June 10, 1984 